\title{
Membrane vesicles derived from Pseudomonas aeruginosa and Shigella flexneri can be integrated into the surfaces of other Gram-negative bacteria
}

\author{
Jagath L. Kadurugamuwa† and Terry J. Beveridge
}

Canadian Bacterial Diseases Network, Department of Microbiology, College of Biological Science, University of Guelph, Guelph, Ontario, Canada N1G 2W1
Author for correspondence: Terry J. Beveridge. Tel: +1 5198244120 ext. 3366. Fax: + 15198371802 . e-mail: tjb@micro.uoguelph.ca

Incubation of intact Salmonella typhi Ty21a, Salmonella enterica serovar Typhimurium (Salmonella typhimurium) aroA or Escherichia coli DH5 $\alpha$ with membrane vesicles (MVs) derived from either Shigella flexneri M90T or Pseudomonas aeruginosa dsp89 resulted in a significant incorporation of vesicle antigens into the outer membrane of the bacteria; each recipient strain possessed a surface mosaic of new Shigella and Pseudomonas antigens intermixed with the native antigens of the Salmonella or Escherichia strains. Electron microscopy of preparations during the integration of vesicle antigens revealed that the MVs rapidly fused with the outer membrane of the host strains. Western blot analysis of host bacteria confirmed the integration of foreign antigens. Quantitative analysis for binding and fusion of antigens using an ELISA showed that approximately 78.7 $\pm 12.8 \mathrm{ng}$ of the Pseudomonas and $67.5 \pm 13.8 \mathrm{ng}$ of the Shigella LPSs ( $\mu \mathrm{g}$ host protein) ${ }^{-1}$ were integrated into the Sal. typhimurium strain. Similar integrations of the Shigella or Pseudomonas vesicles were found with the $E$. coli or Sal. typhi strains. There was no loss of viability in the recipient bacteria after incorporation of the MVs, although vesicle antigens became diluted during continued growth as daughter cells shared the vesicle antigens. The new antigens were highly stable after being incorporated into recipient strains, being able to withstand storage of several months at $4^{\circ} \mathrm{C}$ as well as several cycles of freezing and thawing. Since the recipient bacteria are common vaccine strains, the procedure described here offers a simple efficient means of introducing exogenous surface antigens, in their native form, into the outer membranes of Gram-negative bacteria for possible vaccine use.

Keywords: Gram-negative membrane vesicles, surface antigens, lipopolysaccharide, outer-membrane proteins

\section{INTRODUCTION}

The shedding of membrane vesicles (MVs) during the growth of a large number of Gram-negative bacteria is a common phenomenon (summarized by Kadurugamuwa \& Beveridge, 1997; also see Chatterjee \& Das, 1967; Devoe \& Gilchrist, 1973; Kadurugamuwa \& Beveridge, 1995; Kondo et al., 1993; Wai et al., 1995; Whitmire \&

†Present address: Roche Vitamins Inc., Research and Development, Building 102, B 309, 340 Kingsland Street, Nutley, NJ 07110-1199, USA.

Abbreviations: MV, membrane vesicle; OMP, outer-membrane protein; TEM, transmission electron microscopy.
Garon, 1993; Wispelwey et al., 1989; Zhou et al., 1998). Because MVs arise from the surface of Gram-negative bacteria, possibly as a result of cell wall turnover (Zhou et al., 1998), they consist of outer-membrane and encapsulated periplasmic components (Beveridge \& Kadurugamuwa, 1996), and have strong biological activity (Mayrand \& Holt, 1988). For an opportunistic pathogen such as Pseudomonas aeruginosa which often depends on the Xcp system for secretion (Filloux et al., 1998), MVs could be an important auxiliary form of secretion since they are rich not only in LPS and outermembrane proteins (OMPs), but also in such enzymes as alkaline phosphatase, phospholipase $\mathrm{C}$, protease, 
peptidoglycan hydrolase and proelastase (Kadurugamuwa \& Beveridge, 1995). All of these constituents can be strong antigens. Surprisingly, MVs from $P$. aeruginosa PAO1 contain (virtually) only serotypic, strain-dependent LPS (B-band LPS) and not 'common antigen' LPS (A-band LPS; Kadurugamuwa \& Beveridge, 1995).

Previous research on the major peptidoglycan hydrolase of $P$. aeruginosa PAO1 using synchronized cultures suggested that the enzyme was most active during cell division and that it is most frequently liberated from cells in MVs during the exponential growth phase (Li et al., 1996). These hydrolase-containing MVs can digest the cell walls of other bacteria including both Grampositive and Gram-negative varieties ( $\mathrm{Li}$ et al., 1998; Kadurugamuwa \& Beveridge, 1996), even those covered with an S-layer (Kadurugamuwa et al., 1998). MVs from other Gram-negative bacteria are not as lytic as those from $P$. aeruginosa PAO1 (Li et al., 1998).

While performing this cell lysis research with Gramnegative bacteria, electron microscopy indicated that the exogenous MVs first attached to the outer membrane and then rapidly annealed into it (Kadurugamuwa \& Beveridge, 1996). This phenomenon could have important ramifications since the integration of a foreign MV from a donor bacterium would introduce constituents from the donor directly into the recipient. Interestingly, DNA-containing MVs from one strain of Neisseria gonorrhoeae have the ability to transform other strains (Dorward et al., 1989). If the MVs from a donor bacterium possessed a potent peptidoglycan hydrolase, such as those from $P$. aeruginosa PAO1, it could result in the death of a recipient cell (Kadurugamuwa \& Beveridge, 1996). Yet, if the donor MVs had poor lytic properties (such as those from $P$. aeruginosa dps89 and Shigella flexneri M90T; J. L. Kadurugamuwa \& T. J. Beveridge, unpublished), the host bacterium would not be lysed and the donor components could become firmly integrated into the recipient.

In natural environments containing mixed microbial communities, this interdigitation of donor components by MVs into foreign recipient cells throughout the bacterial population could provide a physical means of communication whereby specific substances are passed from one cell to another. In a more medically applied way, the phenomenon of MV integration into other Gram-negative bacteria could be used in the formulation of novel inexpensive vaccines. For example, the MVs from Gram-negative pathogens could be incorporated into the surfaces of live attenuated vaccine strains such as those derived from Escherichia, Salmonella and Shigella (Curtiss et al., 1989; Lindberg, 1990; Sansonetti \& Arondel, 1989; Germanier \& Fürer, 1975). It is therefore important to clearly demonstrate that MVs from donor strains can be firmly integrated into a variety of recipient bacteria with no ill effects.

Here we report the integration of MVs from $P$. aeruginosa dps89 and Shig. flexneri M90T (as donor strains) into Salmonella typhi Ty21a, Salmonella enterica serovar Typhimurium aroA (herein called Salmonella typhimurium aroA) and Escherichia coli $\mathrm{DH} 5 \alpha$ (as recipient strains). In this way, hybrid recipient cells carrying antigens from the two types of MVs (singly and together) are produced. Since the three recipient strains are from the Enterobacteriaceae and both Sal. typhimurium aroA and Sal. typhi 21a have metabolic defects which inhibit normal growth unless an uncommon but essential nutrient is supplied, they have the potential to be used as oral vaccines (Cárdenas \& Clements, 1992; Cárdenas et al., 1994; Charles \& Dougan, 1990; Cornelis et al., 1996; Darji et al., 1997; Formal et al., 1981; Maskell et al., 1987; Hoiseth \& Stocker, 1981) which circumvents the endotoxic effects of recipient and MV LPS. Of the three recipient bacteria, Sal. typhimurium aroA could be the better vaccine candidate (Cárdenas et al., 1994) and the major proportion of the experimentation in our report concentrates on this strain. Sal. typhi Ty21a and E. coli DH5 $\alpha$ are also incorporated into the study to ensure broader application of the phenomenon and to show that donor MV fusion to host bacteria could be a general phenomenon.

\section{METHODS}

Bacterial strains and growth conditions. P. aeruginosa dps 89 (an A-band LPS defective mutant of the PAO1 strain; Kadurugamuwa et al., 1993), Salmonella typhi Ty21a (Swiss Serum and Vaccine Institute, Berne, Switzerland; Germanier \& Fürer, 1975), Escherichia coli DH5 $\alpha$ (Gibco-BRL), Salmonella enterica serovar Typhimurium (Salmonella typhimurium) aroA strain SL 3261 (Hoiseth \& Stocker, 1981) and Shigella flexneri M90T serotype 5 (Kadurugamuwa et al., 1991) were grown in Trypticase soy broth (TSB) with shaking on an orbital shaker at $37^{\circ} \mathrm{C}$ as described previously by Kadurugamuwa \& Beveridge (1995).

Isolation of MVs. MVs were isolated from exponentially growing cells of dps89 and M90T as outlined previously (Kadurugamuwa \& Beveridge, 1995, 1998). Briefly, cells from 0.51 cultures grown in TSB were removed from suspension by centrifugation at $6000 \mathrm{~g}$ for $15 \mathrm{~min}$. The supernatants were filtered sequentially through 0.45 and $0.22 \mu \mathrm{m}$ pore-sized cellulose acetate membranes (MSI) to remove residual cells. $\mathrm{MVs}$ were removed from the resulting filtrates by centrifugation at $150000 \mathrm{~g}$ for $3 \mathrm{~h}$ at $5{ }^{\circ} \mathrm{C}$ and the vesicle pellet was washed and resuspended in PBS (pH 7.4).

Integration of MVs with bacterial cells. Exponentially growing cultures of Ty21a, SL 3261 and DH $5 \alpha$ in TSB were washed and diluted in PBS ( $\mathrm{pH} 7 \cdot 4$ ) to produce a bacterial suspension of $1 \times 10^{*}$ c.f.u. ml ${ }^{1}$. These cells were mixed $4: 1$ with a suspension of MVs (each MV preparation contained 2.5$100 \mu \mathrm{g}$ protein $\mathrm{ml}^{-1}$ ) from either strain dps89 or M90T, or a mixture from both $\left(100 \mu \mathrm{g}\right.$ protein $\mathrm{ml}^{-1}$ each), and incubated at $37^{\circ} \mathrm{C}$ for $15 \mathrm{~min}$. The unbound MVs were removed from the cells by centrifuging the cell-MV suspension at $6000 \mathrm{~g}$ for $15 \mathrm{~min}$. This was followed by resuspending the pellet in PBS and sequential filtration through 0.45 and $0.22 \mu \mathrm{m}$ pore-sized cellulose acetate membranes. Finally, $10 \mathrm{ml}$ PBS was passed through the filters to wash the hybrid recipient cells and remove any remaining unbound $M V s$ which had not integrated into the recipient strain. Hybrid cells were collected 
from membrane filters by vortexing the filters in $10-15 \mathrm{ml}$ PBS and centrifugation of the cell suspension at $6000 \mathrm{~g}$ for $15 \mathrm{~min}$. Samples were examined by transmission electron microscopy (TEM) to confirm complete removal of nonintegrated MVs from the cell suspension.

Assay for attachment of MVs to bacterial cells. A combination of methods was used to monitor all aspects of integration of MVs with the recipient bacteria.

(a) ELISA. Quantification of MV LPS antigens integrated with bacterial cells was achieved by performing ELISA (Crowther, 1995). Briefly, 96-well polystyrene flat-bottom microtitre plates (Nunc; Fisher Scientific) were coated with purified LPS $\left(5 \mu \mathrm{g} \mathrm{m}^{-1} ; 100 \mu \mathrm{l}\right.$ per well) (Kadurugamuwa et al., 1991) from M90T, dps89 or SL 3261 as the solid-phase specific antigens. The optimal concentration of LPS and corresponding antibody titre were determined by checkerboard titration. The foreign LPS antigen concentration in hybrid strains was interpolated from a standard curve. Polyclonal antibodies to the purified LPS from Shig. flexneri M90T $(1: 200)$ and mAbs for $P$. aeruginosa dps89 B-band serotype 05 LPS (1:10) were diluted in PBS and the amount of antibody-specific antigen in hybrid strains was determined with alkaline-phosphataseconjugated goat anti-rabbit $\operatorname{IgG}(1: 3000)$ and goat anti-mouse IgM (1:3000) (Cedarlane) with $p$-nitrophenyl phosphate (Bio$\mathrm{Rad}$ ) as the substrate. Background non-specific activity was determined by testing the samples in uncoated wells lacking either the primary and/or secondary antibody; the background absorbance was subtracted from the measured absorbance to provide the antigen-specific increase in absorbance. The results refer to the mean values obtained from samples of six hybrids constructed on separate days. Standard deviations represent variations between individual samples. Experiments were performed to test sensitivity, specificity, precision and accuracy of assay. The limits of detection were $0 \cdot 2-2 \mu \mathrm{g} \mathrm{ml}^{-1}$. Intra-run (coefficient of variation $=3.3 \%$ ) and inter-run (coefficient of variation $=15.5 \%$ ) variations were calculated and were always comparable to those of similar assays. The assay's correlation coefficient $(r$ $=0.9971-0.9998)$ demonstrated a statistically significant correlation between the absorbance of the sample and the concentration of LPS antigen. Similar trends were found when Ty21a and DH5 $\alpha$ were used as recipient strains.

(b) Western blotting. A $40 \mu \mathrm{g}$ sample of protein was digested with proteinase $\mathrm{K}\left(10 \mu \mathrm{g} \mathrm{m}^{-1}\right)$ and the sample separated by SDS-PAGE. This gel was transferred onto nitrocellulose and the integrated antigens in recipient strains were detected by incubation with either mAbs for dps89 B-band LPS (Kadurugamuwa et al., 1993) or polyclonal antibodies to the purified LPS from M90T (Kadurugamuwa et al., 1991).

(c) Immunogold labelling. The fusion of MVs from M90T and dps89 with SL 3261 , DH5 $\alpha$ or Ty21a was demonstrated by immunogold labelling of whole mounts and thin sections directly on Formvar- and carbon-coated nickel TEM grids using LPS-specific polyclonal antibodies to M90T or mAbs to dps89 LPS. The simultaneous detection of antigens from the MVs of the two separate pathogens in hybrid strains was carried out by a double immunogold staining method using different-sized gold particles. Whole mounts and thin sections were labelled using the same technique except that the thinsectioned specimens were first fixed. Briefly, these samples were enrobed in $2 \%(\mathrm{w} / \mathrm{v})$ molten Noble agar, fixed with a solution containing $0 \cdot 1 \%(\mathrm{v} / \mathrm{v})$ glutaraldehyde and $2 \%(\mathrm{v} / \mathrm{v})$ formaldehyde in $50 \mathrm{mM}$ HEPES (Sigma) buffer ( $\mathrm{pH} 6.8$ ) for $1 \mathrm{~h}$ at $4^{\circ} \mathrm{C}$. After this mild fixation, an LR White (Marivac) embedding regimen was performed (Kadurugamuwa \&
Beveridge, 1995). After the plastic had hardened, thin sections were cut and incubated in $0.01 \mathrm{MPBS}(\mathrm{pH} \mathrm{7.2)}$ containing $1 \%$ $(\mathrm{w} / \mathrm{v})$ BSA and $0.1 \%(\mathrm{v} / \mathrm{v})$ Tween 20 (PBS-BSA-T) for $5 \mathrm{~min}$ followed by another $5 \mathrm{~min}$ incubation in PBS containing $0.02 \mathrm{M}$ glycine. Next, the sections were incubated for $10 \mathrm{~min}$ in PBS containing $2 \%(\mathrm{w} / \mathrm{v})$ skimmed milk at room temperature. Grids were then incubated with LPS-specific polyclonal antibodies to M90T $(1 / 100)$ or mAbs to dps89 LPS $(1 / 10)$ in PBS-BSA-T for $1 \mathrm{~h}$ at room temperature. Afterwards, the grids were rinsed in PBS-BSA and incubated in a colloidal gold mixture containing particles conjugated to protein $A$ $(5 \mathrm{~nm}$ in diameter; protein $\mathrm{A}$-gold) and particles conjugated to anti-mouse $\operatorname{IgM}(15 \mathrm{~nm}$ in diameter; IgM-gold) (EY Laboratories). Both commercial products were diluted 1:100 in PBS-BSA-T and the grids were incubated for $1 \mathrm{~h}$. The grids were washed in PBS-BSA for $5 \mathrm{~min}$ at room temperature, stained with uranyl acetate and lead citrate to contrast the specimen for TEM, and examined with a Philips EM300 microscope operating under standard conditions with the liquid nitrogen cold trap in place. Appropriate positive and negative controls were incorporated into all labelling experiments.

SDS-PAGE. OMPs were prepared from bacterial cell pellets by breaking them with five $60 \mathrm{~s}$ pulses of sonication in an ice bath separated by $30 \mathrm{~s}$ intervals for cooling. Unbroken cells were removed by centrifugation at $6000 \mathrm{~g}$ for $10 \mathrm{~min}$. Sarkosyl ( $\mathrm{N}-$ lauroylsarcosine, sodium salt; Sigma) was added to the lysate to a final concentration of $2 \%(\mathrm{w} / \mathrm{v})$ and the mixture was incubated for $30 \mathrm{~min}$ at room temperature to dissolve plasma membrane fragments. Then the mixture was centrifuged at $38000 \mathrm{~g}$ for $1 \mathrm{~h}$, and the membrane pellet was washed twice with distilled water, resuspended in a small volume of distilled water, and stored at $-20^{\circ} \mathrm{C}$. MVs, OMPs and whole cells were boiled for $10 \mathrm{~min}$ in sample buffer (containing $60 \mathrm{mM}$ Tris, $\mathrm{pH} 6.8 ; 10 \%, \mathrm{v} / \mathrm{v}$, glycerol ; $2 \%$, w/v, SDS $0.05 \%$, $\mathrm{w} / \mathrm{v}$, bromophenol blue; and $1 \mu \mathrm{l} 2 \%, \mathrm{v} / \mathrm{v}, \beta$-mercaptoethanol) and resolved in a $13 \%(\mathrm{w} / \mathrm{v})$ polyacrylamide gel stained with Coomassie brilliant blue (Kadurugamuwa \& Beveridge, 1995).

Viability of MV-treated strains. Viability was determined by plate count on Trypticase soy agar (TSA) and by monitoring the increase in $\mathrm{OD}_{600}$ during growth in TSB after MVs from dps89 and M90T had been incorporated into SL3261, Ty21a and $\mathrm{DH} 5 \alpha$ as compared to untreated control cultures.

\section{RESULTS}

\section{TEM and SDS-PAGE analyses of MVs}

By electron microscopy of thin sections, isolated and purified MVs from Shig. flexneri M90T and P. aeruginosa dps89 were shown to be bilayered spherical vesicles approximately $80 \mathrm{~nm}$ in diameter as shown previously (Kadurugamuwa \& Beveridge, 1995, 1998). The protein profiles of whole cell lysates, OMPs (extracted from whole cells) and MVs from M90T and dps89 were compared by SDS-PAGE (Fig. 1a). The banding patterns of M90T MVs were similar, but not identical, to the corresponding OMPs from whole cells; major $\sim 35$ and $37 \mathrm{kDa}$ bands were prominent with trace amounts of two additional proteins of $\sim 70$ and $97 \mathrm{kDa}$ (Kadurugamuwa \& Beveridge, 1998). The prominently stained MV bands from dps89 included $\sim 45$ and $55 \mathrm{kDa}$ proteins, which were also prominent in the OMP fraction. The intensely stained $38 \mathrm{kDa}$ protein 
(a)

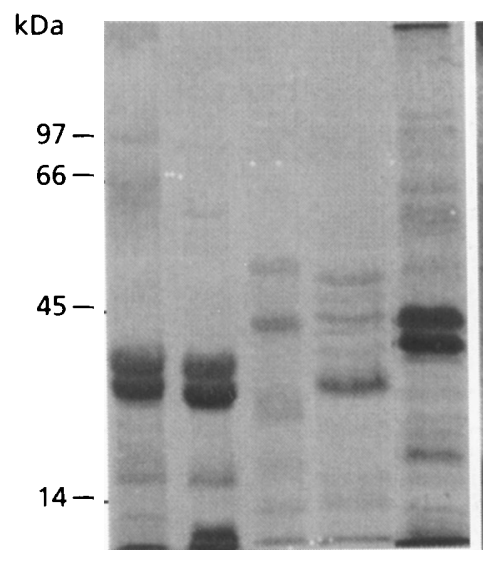

(b)

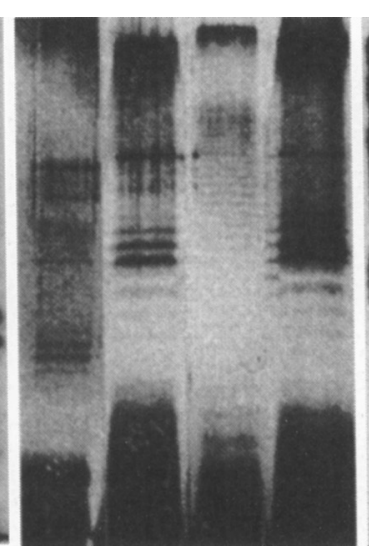

(c)

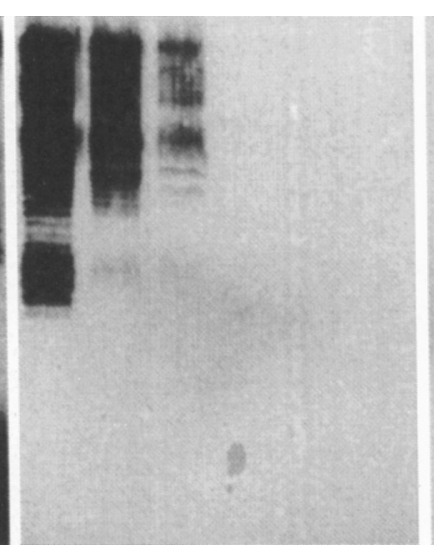

(d)

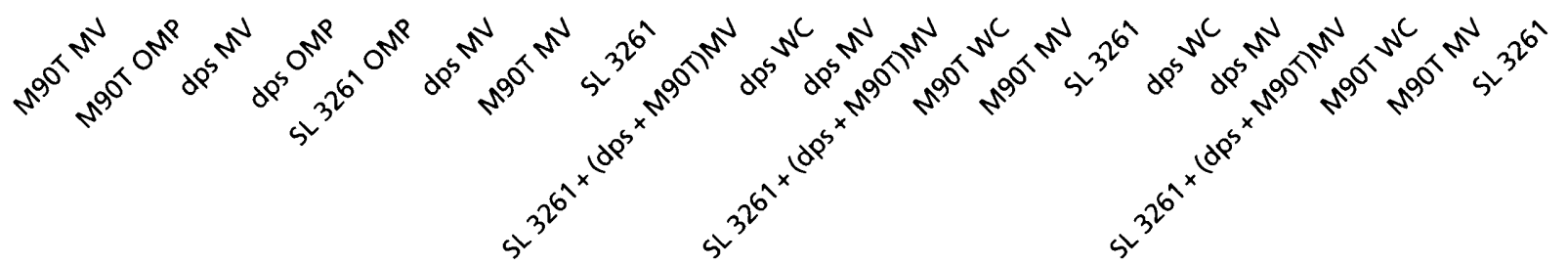

Fig. 1. (a) SDS-PAGE protein profiles of M90T and dps 89 MVs, and M90T, dps 89 and SL 3261 OMPs. Each lane contains $25 \mathrm{\mu g}$ protein. Numbers in the left margin denote molecular mass. (b) Silver-stained SDS-PAGE of LPS from dps 89 and M90T MVs and SL 3261 whole cells before and after integration of dps89 and M90T MVs. (c, d) Western blots to demonstrate and identify the MV antigens that were integrated into SL 3261. The LPS that was fractionated by SDS-PAGE was transferred onto nitrocellulose and reacted with mAbs specific for dps89 LPS (c) and against antibodies specific to the LPS of M90T (d). Neither of the antibodies reacted against the LPS of SL 3261. M90T-specific antibodies did not cross-react with dps89 nor did dps89-specific antibodies cross-react with M90T LPS. WC denotes whole cells.

band seen in the dps 89 OMP sample was barely detected in dps89 MVs. The major OMPs in the Salmonella strain appeared to be in the $\sim 38-42 \mathrm{kDa}$ region.

\section{SDS-PAGE banding pattern and immunoreactivities of integrated LPS}

LPS samples were separated by SDS-PAGE and silverstained to characterize carbohydrate moieties and their banding patterns. The banding patterns of LPS from dps89 and M90T MVs and from whole cells of SL 3261 are shown in Fig. 1(b). Both types of MVs showed characteristic LPS ladder-like bands and core regions with relative mobilities similar to those seen in whole cells (Kadurugamuwa \& Beveridge, 1995; Sansonetti \& Arondel, 1989). The banding pattern of LPS in SL 3261 following fusion and integration of both types of $\mathrm{MVs}$ altered its typical banding pattern. The distinct separation between the SL 3261 bands was no longer present since those of dps89 and M90T MVs were intermixed between them; i.e. the co-expression of LPSs from the MVs with SL 3261 LPS in the recipient's outer membrane. To identify M90T and dps89 LPS antigens from the surface of SL 3261, electrophoretic blots of LPS from the SDS-PAGE gel described in Fig. 1(b) were reacted with either dps89-specific or M90T-specific LPS antisera (Fig. 1c, d). The banding pattern and immunoreactivities of the integrated dps89 LPS (into SL 3261) were subtly different to those seen from dps89 whole cells or MVs alone (Fig. 1c). The high-molecular-mass portion of the ladder-like banding pattern in immunoblots reacted most strongly with dps89-specific antibodies. A difference was also detected with integrated M90T LPS, but here the middle to upper portion reacted with M90T-specific antibodies (Fig. 1d). This suggested an integration and fusion of LPS from MVs into the outer membrane of SL 3261 (Fig. 1c, d). M90T or dps89 anti-LPS antibodies did not react with control SL 3261 cells which were not treated with MVs (Fig. 1c, d). M90T-specific antibodies did not cross-react with dps89 antigens nor dps89-specific antibodies with M90T antigens (Fig. 1c, d). The LPSs of M90T or dps89 MVs reacted similarly with type-specific antibodies after integration into either of the $\mathrm{DH} 5 \alpha$ or Ty21a strains (data not shown).

\section{Fusion and integration of MV antigens into recipient bacteria}

The firm integration of M90T and dps89 MV LPS antigens into SL 3261, Ty21a or DH5 $\alpha$ was confirmed using anti-LPS antibodies and TEM. Immunogold labelling of MV-treated bacteria with LPS-specific antibodies illustrated a good topographic distribution of attached MV antigens on the host cell surface by both whole mount (Fig. 2b, c) and thin-sectioning (Fig. 2d-f) tech- 


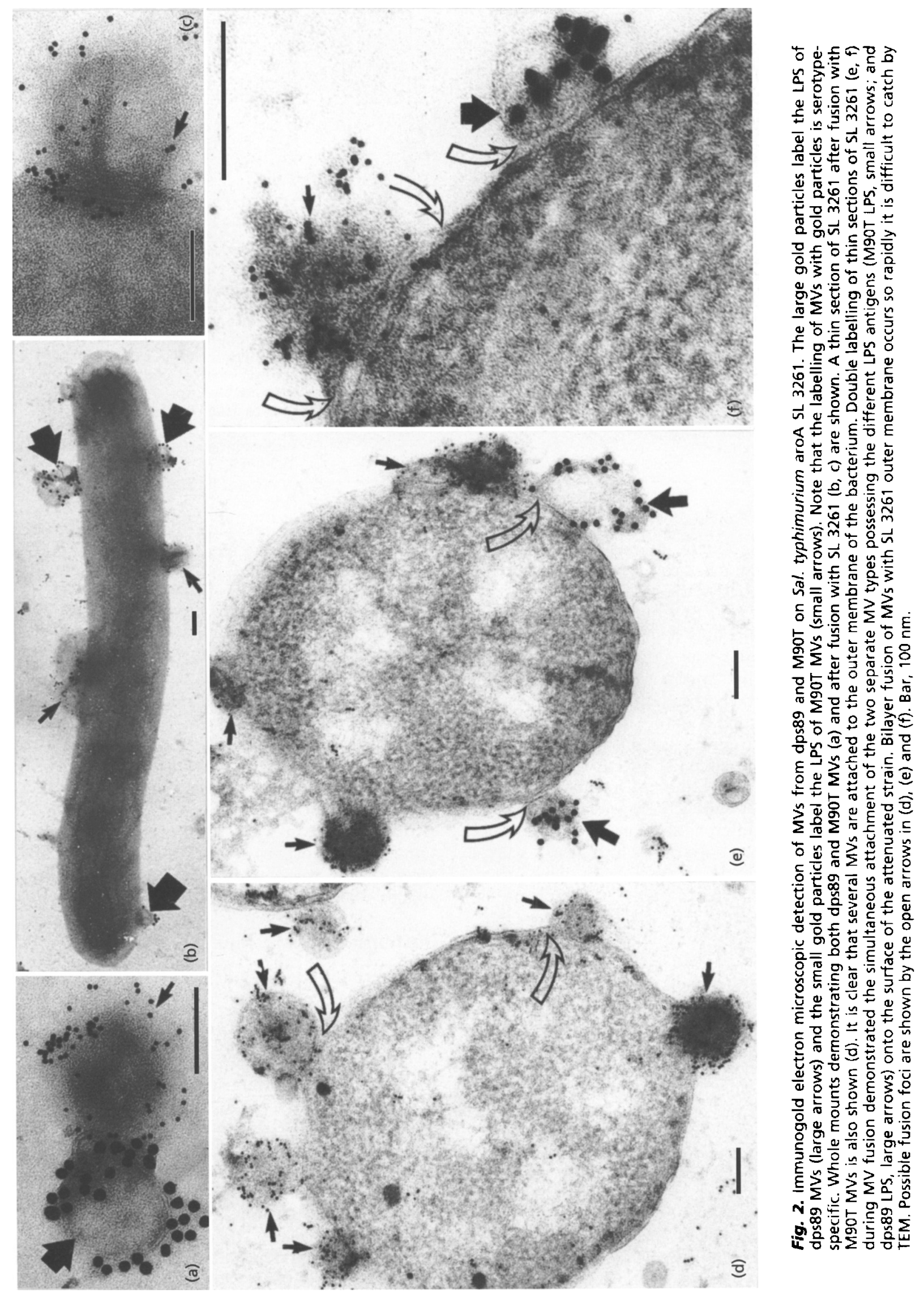




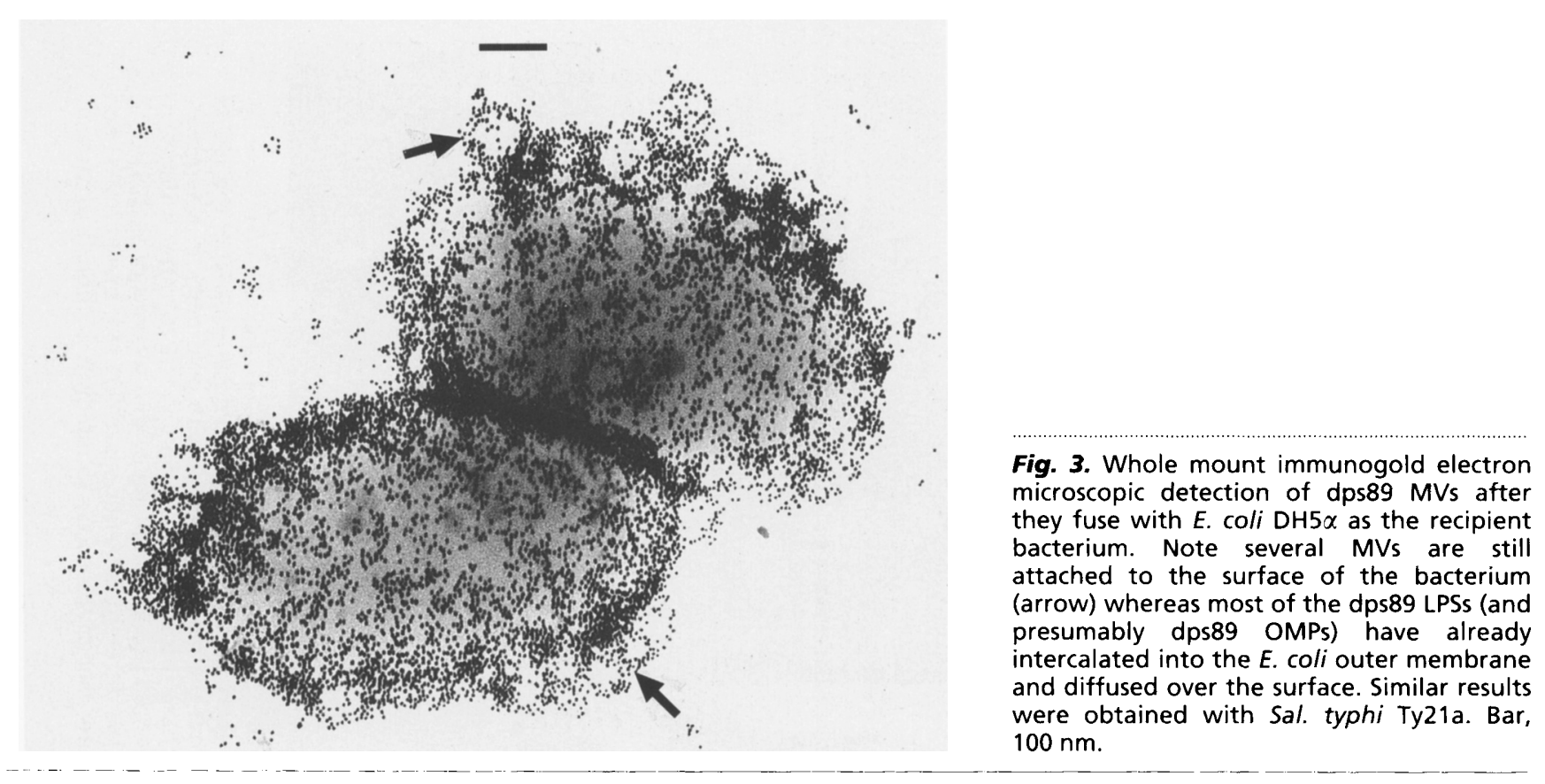

niques. Using the whole mount technique, it was obvious that both types of MVs attached to the outer membrane of a recipient cell and that MV LPS entered the cell's outer membrane (Fig. 2b). By implication, MV integration into (recipient) outer membrane must occur but the actual fusion point was difficult to capture by TEM. Presumably, fusion was so rapid after initial attachment that it was rarely seen (Fig. 2c; Zimmerberg et al., 1993). Thin sectioning is a more definitive method of discerning the fusion event, but only a $\sim 600-\mathrm{nm}$ thick slice of a bacterium is seen at a time and this low sampling level makes the likelihood of capturing fusion even rarer. In Fig. 2(d-f), possible fusion sites are shown. Double labelling using different-sized gold particles for each MV LPS proved that both dps89 and M90T were together integrated with the outer membrane of the recipient cells when both types of donor MVs were used at the same time (Fig. 2e, f). Fig. 3 demonstrates the integration of dps89 MVs with DH5 $\alpha$, and similar results were also seen with Ty21a as the recipient. Both of these recipient strains could also integrate the two MV types at the same time. Although we have immunogold-labelled only distinct LPS in all TEM preparations, it is presumed that other MV constituents (i.e. OMPs) are also integrated as was suggested by the previous SDS-PAGE results (Fig. 1a). The integration of MVs into the surface of other Gram-negative bacteria and with human gut epithelial cells has also been demonstrated in previous studies (Kadurugamuwa \& Beveridge, 1996, 1998).

After the MVs had been integrated into recipient cells, the bacteria resumed growth after a brief lag period $(15-20 \mathrm{~min})$ when they were resuspended in TSB (see subsequent 'cell viability' section for more details). Immunogold TEM of these hybrid cells showed that, as growth proceeded, the incidence of the initial labelling decreased. It was apparent that the foreign integrated antigens were being shared between dividing daughter cells and, eventually, diluted to low incidences over time. If there was no growth, the foreign antigens remained firmly embedded in the surfaces of all recipient strains. They could still be labelled by the Western and TEM techniques after vigorous shaking, several months of storage in a refrigerator at $4{ }^{\circ} \mathrm{C}$, or after several cycles of freezing and thawing the treated cells. In fact, the $M V s$ on their own (without integration) were also stable in suspension. TEM of negative stains of these MVs showed that they maintained the same vesicle diameter during storage or after the freeze/thaw cycling; presumably this must be the most thermodynamically stable form of the vesicles (Lauffer, 1989; Lakshminarayanaiah, 1984).

\section{Quantification of foreign antigens in recipient cells}

To determine the amount of foreign antigens that was incorporated into recipient cells following our fusion protocol, we estimated the serotype-specific LPS antigens in the hybrid bacteria by ELISA. MV antigen incorporation appeared to be concentration-dependent (Table 1); as the concentration of either dps89 or M90T MVs was increased from 25 to $100 \mu \mathrm{g}$ protein $\mathrm{ml}^{-1}$, the incorporation of antigen into SL 3261 dramatically increased. Incubation of SL 3261 with either type of MVs at concentrations above $100 \mu \mathrm{g} \mathrm{m}^{-1}$ did not cause any further significant increase in antigen incorporation, suggesting that there is a saturation point for the amount of this foreign matter that a cell can complex into its outer membrane. This is further illustrated when both dps89 and M90T MVs were added (each at $100 \mu \mathrm{g} \mathrm{ml}^{-1}$ ) simultaneously (Table 1). In this case, the total amount 
Table 1. Quantitative measurement of MV antigens in SL 3261

\begin{tabular}{|lccc|}
\hline MV type & Recipient cell & Amount added & Amount transferred $\dagger$ \\
\hline M90T & SL 3261 & 25 & $2 \cdot 0 \pm 1 \cdot 9$ \\
& & 50 & $13 \cdot 0 \pm 7 \cdot 1$ \\
& & 100 & $67 \cdot 5 \pm 17 \cdot 8$ \\
dps89 & 25 & $8 \cdot 2 \pm 1 \cdot 9$ \\
& & 50 & $31 \cdot 7 \pm 10 \cdot 2$ \\
M90T + dps89 & 100 & $78 \cdot 7 \pm 12 \cdot 8$ \\
& & $100($ each) & $33 \cdot 6 \pm 4 \cdot 6(\mathrm{M} 90 \mathrm{~T})$ \\
& SL 3261 & 100 (each) & $38 \cdot 6 \pm 5 \cdot 8(\mathrm{dps} 89)$ \\
\hline
\end{tabular}

* $\mu \mathrm{g} \mathrm{MV} \mathrm{protein} \mathrm{ml}^{-1}$.

†ng type-specific LPS per $\mu \mathrm{g}$ total protein from recipient cells.

of dps89 plus M90T LPS which was integrated into SL 3261 [ 72 ng LPS ( $\mu$ g SL 3261 protein) ${ }^{-1}$ ] was approximately equivalent to either of the MVs when they were added alone [i.e. 78.7 and $67.5 \mathrm{ng}$ LPS ( $\mu \mathrm{g}$ SL 3261 protein $)^{-1}$ for dps 89 and $\mathrm{M} 90 \mathrm{~T}$, respectively]. In general, it was evident that MVs derived from $P$. aeruginosa were slightly more efficient at integrating with SL 3261 than those from Shig. flexneri (Table 1). This trend was also true when DH5 $\alpha$ or Ty21a was used as recipient cells. This presumably reflects a subtle difference in the fusion compatibility of each type of $M V$ with the outer membrane of the recipient cell. After fusion, the components of each MV would eventually mix with the pre-existing outer-membrane constituents of the recipient cell and diffuse (laterally) around the bacterium (Mouritsen \& Bloom, 1993). Although the MV components are also derived from a (foreign) outer membrane and are therefore analogous to that of the recipient, their different chemical composition could affect membrane fluidity, permeability and other characteristics. It is quite possible that only a certain amount of foreign matter would be tolerated by the recipient and the degree of foreignness would be a reflection of the chemical composition of the donor MV.

\section{Cell viability}

To determine whether the fusion protocol had a deleterious effect on recipient cells, we monitored their viability following incubation with MVs. Results indicated that fusion of foreign antigens derived from either dps89 or M90T had no detectable effects on cell viability. More than $90 \%$ of the cells remained viable as determined by colony counts on TSA plates after incubation with either dps89 or M90T MVs, or with both types together. Control cells treated in an identical manner (but without MVs) showed similar viability differences, indicating that the small percentage in viability loss was most likely due to the various experimental protocols that the cells were subjected to during sample preparation. When cells were grown in broth (TSB), control and MV-treated cells of SL 3261, Ty21a and DH5 $\alpha$ had similar lag times and growth phases. As mentioned before, the integrated MVs became 'diluted' as the cells grew and divided.

\section{DISCUSSION}

The release of MVs from Gram-negative bacteria is a natural phenomenon which is seen in both laboratorygrown bacteria and natural populations of microorganisms (Beveridge et al., 1997). It is difficult to imagine that such a wide-range, common shedding of vesicles would be purposeless. MVs are expensive in cellular material (that could be recycled), encompass periplasmic substance in a protective membranous bilayer, have enzymic activity, and possess potentially strong antigens (Kadurugamuwa \& Beveridge, 1997). These attributes argue strongly for one (or more) common function for these natural particles of cellular substance. We have suggested that at least one function could be for the delivery of cellular materials; MVs could be considered to be an alternative secretion pathway (Kadurugamuwa \& Beveridge, 1995) in which secretion substances are protected from exogenous factors (such as hydrolases) by the membrane bilayer. One could envision MVs as small delivery vehicles sent from one cell to another in order to physically convey materials from donor to recipient, especially since the surface of the MV should be readily compatible with other membrane surfaces.

For this delivery concept to be correct, it is important to provide convincing evidence that donor MVs are actually integrated into the recipient's surface. In this way, as MVs fuse into the cell's outer membrane, the lumenal contents of the MVs would be forced into the recipient's periplasm and a hybrid membrane (consisting of donor MV membrane and recipient outer membrane) would be formed (see Kadurugamuwa \& Beveridge, 1996, for postulated details). Previous experimentation from our laboratory has provided preliminary evidence suggesting that the delivery concept is correct since MVs containing a potent peptidoglycan hydrolase can lyse Gramnegative bacteria (Kadurugamuwa \& Beveridge, 1996) but, since they also lyse Gram-positive cells, this evidence is not definitive. Also, MVs which have been 
manipulated so that they contain gentamicin (g-MVs) were able to convey the antibiotic to a mammalian cell line which had been intracellularly infected with Shig. flexneri, and eventually killed this invasive pathogen (Kadurugamuwa \& Beveridge, 1998), which indirectly suggests a delivery function for MVs. In this current report, we provide definitive evidence showing the integration of surface antigens from two donor MVs into the outer membrane of three separate Gramnegative species as recipient cells. This argues strongly that MVs have the potential to deliver donor materials into recipient cells. Together with our mounting evidence that the shedding of MVs from Gram-negative bacteria is a common occurrence which spans many such eubacteria, it is probable that this is an important common route of physical communication between bacteria. The substances which are conveyed could be deleterious to the recipient (Kadurugamuwa \& Beveridge, 1996; Li et al., 1998), advantageous (Dorward et al., 1989) or possibly actual signal substances (e.g. agents similar to pheromones). It is our hope that our present results will stimulate research to better illuminate the substances which MVs deliver.

The exact mechanism by which an MV is produced and the mechanism by which an MV fuses into a recipient cell has been difficult to study because this is a nanoscale process occurring within complicated surfaces of minute cells. Yet, some assumptions can be made from the available data. Since LPS remains on the outer face of an MV (i.e. an MV is 'right-side-out'; Kadurugamuwa \& Beveridge, 1995) and since MVs have a full complement of OMPs, no drastic rearrangement of outer-membrane constituents can be taking place during MV formation. Once the MV has formed and escaped from the donor bacterium, it must be in its low-energy structural form and would be energetically stable (Lauffer, 1989; Lakshminarayanaiah, 1984). This is why MVs withstand long storage under refrigeration. It is also why they can be subjected to repeated freezing and thawing (Kadurugamuwa \& Beveridge, 1997); even if they are punctured by small ice crystals during freezing they are thermodynamically forced back into their low-energy intact vesicle form on thawing, ensuring that their contents are still enclosed and protected.

When MVs are mixed with a recipient strain of a bacterium, a proportion of the MVs would continuously impact with the recipient's outer surface, thereby affecting the energy load of the vesicle. This would be especially true because the recipient's surface is a bilayered membrane complete with an analogous LPS on its outer face. The divalent metal distribution between MV and outer membrane could rapidly change and the innate lipid fluidity of the two bilayers should cause fusion (Kadurugamuwa \& Beveridge, 1996). Indeed, as this present report shows, there is a rapid and high degree of MV fusion into the foreign recipient membrane.

One obvious medical application of antigen transfer by MVs would be as a vaccine delivery system for mucosal immunity using the incorporation of foreign 'pathogenic' antigens into live attenuated carrier strains such as the Salmonella or Escherichia spp. used in this study (Cárdenas \& Clements, 1992; Charles \& Dougan, 1990; Levine et al., 1989; Roberts et al., 1994; Stocker, 1990). These hybrid strains are particularly attractive because they can be given orally and do not replicate in mammalian tissue, but they can still invade and deliver foreign antigens to elicit secretory, humoral and cellmediated immune responses to the new antigens (Cárdenas \& Clements, 1992; Georgiou et al., 1997; Stocker, 1990; Su et al., 1992). Considerable effort is being made to use recombinant DNA techniques to express protective antigens such as LPS and proteins from pathogenic bacteria in such live, attenuated carrier strains (Cárdenas \& Clements, 1992; Charles \& Dougan, 1990; Curtiss et al., 1989; Formal et al., 1981; Mahan et al., 1992; Maskell et al., 1987; Roberts et al., 1994; Su et al., 1992). However, the use of genetic procedures to transfer foreign genes into attenuated strains has several disadvantages. For example, there is the necessity of developing constitutive expression systems, the possibility of the unwanted environmental release of recombinant bacteria, the unexpected expression of antibiotic resistance following strain construction, the eventual reversion to virulence over time, the poor surface expression of improperly folded inactive forms of antigens for recognition by the immune system, and the instability of the cloned genes (Charles \& Dougan, 1990; Cornelis et al., 1996; Fuchs et al., 1991; Georgiou et al., 1996; Hofnung, 1991; Laukkanen et al., 1993). Although it has been attempted to make transformants more stable by introducing the cloned genes into the chromosome of the carrier strain, the level of expression of foreign antigens seems to be lower than that when expressed by a plasmid. In our present report, we have taken a novel, yet simple, approach to alleviate these problems and it does not require a genetic manipulation. The natural ability of $\mathrm{MVs}$ from one bacterium to adhere and fuse with another was employed to transfer surface antigens of pathogenic bacteria into the surface of attenuated vaccine strains. In fact, our protocol should allow any macromolecule that is expressed on a Gram-negative bacterial surface, or in its periplasm, to be captured and delivered by an MV. Such macromolecules could include OMPs, pilin, smaller peptides, LPS, phospholipids or enzymes (Kadurugamuwa \& Beveridge, 1995, 1996, 1998). Since MVs are commonly produced by many Gram-negative bacteria, it should be possible to produce a full spectrum of antigens from a number of different pathogens (including up-to-date serotypes or antigenic variants) by fusion of MVs to produce a heterologous multivalentantigen carrier strain. However, since the integrated antigens are not produced as an integral part of the carrier strain by its own genetic machinery, normal cell growth and outer-membrane turnover would eventually dilute out our MV expression system (as seen in our growth studies in this report). This is one of the reasons why we chose to incorporate MVs into strains possessing metabolic defects, such as the Sal. typhi Ty21a and Sal. 
typhimurium aroA. These live attenuated vaccine strains cannot grow unless the proper metabolite is supplied to them, but they remain viable. Accordingly, once our MVs have been incorporated into these metabolically defective strains, MV antigenic components would be stable and continuously expressed. Once these carrier strains are in the host tissue, they stop growing and outer-membrane turnover is arrested (Cárdenas \& Clements, 1992; Charles \& Dougan, 1990; Georgiou et al., 1997; Roberts et al., 1994; Stocker, 1990); integrated antigens are not released or replaced.

It is well-documented that the critical event determining the development of a humoral immune response against foreign antigens delivered by a Salmonella vector is the initial amount of antigen that primes the gut-associated lymphoid tissue, rather than the persistence of the vaccine strain in the tissue (Cárdenas \& Clements, 1992; Cárdenas et al., 1994). The MV fusion technique may have even broader applicability as a method for rapid screening and selecting immunogenic antigens for vaccine development. Since the construction of hybrid recipient strains is rapid, the identification and selection of suitable antigen candidates would be cost-effective prior to initiating a large-scale immunization.

\section{ACKNOWLEDGEMENTS}

J. S. Lam provided us with $\mathrm{mAbs}$ to $P$. aeruginosa LPS and P. J. Sansonetti the M90T strain. We thank Anuradha Saxena and Dianne Moyles for their technical assistance with this study. This work was made possible by funding through the Canadian Bacterial Disease Network as a National Center of Excellence. The electron microscopy was performed in the NSERC Guelph Regional STEM Facility, which is partially funded by a Natural Sciences and Engineering Research Council of Canada (NSERC) Major Facilities Access grant to T.J.B.

\section{REFERENCES}

Beveridge, T. J. \& Kadurugamuwa, J. L. (1996). Periplasm, periplasmic spaces, and their relation to bacterial wall structure: novel secretion of selected periplasmic proteins from Pseudomonas aeruginosa. Microb Drug Resist 2, 1-8.

Beveridge, T. J., Makin, A. S., Kadurugamuwa, J. L. \& Li, Z. (1997). Interactions between biofilms and the environment. FEMS Microbiol Rev 20, 291-303.

Cárdenas, L. \& Clements, J. D. (1992). Oral immunization using live attenuated Salmonella spp. as carriers of foreign antigens. Clin Microbiol Rev 5, 328-342.

Cárdenas, L., Dasgupta, U. \& Clements, J. D. (1994). Influence of strain viability and antigen dose on the use of attenuated mutants of Salmonella as vaccine carriers. Vaccine 12, 833-840.

Charles, I. \& Dougan, G. (1990). Gene expression and the development of live enteric vaccines. Trends Biotechnol 8, 117-121.

Chatterjee, S. N. \& Das, J. (1967). Electron microscopic observations on the excretion of cell-wall material by Vibrio cholerae. J Gen Microbiol 49, 1-11.

Cornelis, P., Sierra, J. C., Lim, A., Jr \& 9 other authors (1996). Development of new cloning vectors for the production of immunogenic outer membrane fusion proteins in Escherichia coli. BioTechnology 14, 203-208.

Crowther, J. R. (1995). ELISA. Theory and practice. Methods Mol Biol 42, 131-149.

Curtiss, R., Nakayama, K. \& Kelly, S. M. (1989). Recombinant avirulent Salmonella vaccine strain with stable maintenance and high level expression of cloned genes in vivo. Immunol Investig 18, 583-596.

Darji, A., Guzmán, C. A., Gerstel, B., Wachholz, P., Timmis, K. N., Wehland, J., Chakraborty, T. \& Weiss, S. (1997). Oral somatic transgene vaccination using attenuated $S$. typhimurium. Cell 91, $765-775$.

Devoe, I. W. \& Gilchrist, J. E. (1973). Release of endotoxin in the form of cell wall blebs during in vitro growth of Neisseria meningitidis. J Exp Med 138, 1156-1166.

Dorward, D. W., Garon, C. F. \& Judd, R. C. (1989). Export and intercellular transfer of DNA via membrane blebs of Neisseria gonorrboeae. J Bacteriol 171, 2499-2505.

Filloux, A., Michel, G. \& Bally, M. (1998). GSP-dependent protein secretion in Gram-negative bacteria: the Xcp system of Pseudomonas aeruginosa. FEMS Microbiol Rev 22, 177-198.

Formal, S. B., Baron, L. S., Kopecko, D. J., Washington, O., Powell, C. \& Life, C. A. (1981). Construction of a potential bivalent vaccine strain: introduction of Shigella sonnei form 1 antigen genes into the galE Salmonella typhi 21 a typhoid vaccine strain. Infect Immun 34, 746-750.

Fuchs, P., Breitling, F., Dubel, S., Seehaus, T. \& Little, M. (1991). Targeting recombinant antibodies to the surface of Escherichia coli: fusion to a peptidoglycan-associated lipoprotein. BioTechnology 9, 1369-1372.

Georgiou, G., Stephens, D. L., Stathopoulos, C., Poetschle, H. L., Mendenhall, J. \& Earhart, C. F. (1996). Display of $\beta$-lactamase on the Escherichia coli surface: outer membrane phenotypes conferred by Lpp-OmpA- $\beta$-lactamase fusions. Protein Eng 9, 239-247.

Georgiou, G., Stathopoulos, C., Daugherty, P. S., Nayak, A. R., Iverson, B. L. \& Curtiss, R. (1997). Display of heterologous proteins on the surface of microorganisms: from the screening of combinatorial libraries to live recombinant vaccines. Nat Biotechnol 15, 29-34.

Germanier, R. \& Furer, E. (1975). Isolation and characterization of Salmonella typhigal E mutant Ty21a: a candidate strain for a live oral typhoid vaccine. J Infect Dis 131, 553-558.

Hofnung, M. (1991). Expression of foreign polypeptides at the Escherichia coli cell surface. Methods Cell Biol 34, 77-105.

Hoiseth, S. K. \& Stocker, B. A. D. (1981). Aromatic-dependent Salmonella typhimurium are non-virulent and effective as live vaccines. Nature 291, 238-239.

Kadurugamuwa, J. L. \& Beveridge, T. J. (1995). Virulence factors are released from Pseudomonas aeruginosa in association with membrane vesicles during normal growth and exposure to gentamicin: a novel mechanism of enzyme secretion. J Bacteriol 177, 3998-4008.

Kadurugamuwa, J. L. \& Beveridge, T. J. (1996). Bacteriolytic effect of membrane vesicles from Pseudomonas aeruginosa on other bacteria including pathogens: conceptually new antibiotics. J Bacteriol 178, 2767-2774.

Kadurugamuwa, J. L. \& Beveridge, T. J. (1997). Natural release of virulence factors in membrane vesicles by Pseudomonas aeruginosa and the effect of aminoglycoside antibiotics on their release. I Antimicrob Chemother 40, 615-621.

Kadurugamuwa, J. L. \& Beveridge, T. J. (1998). Delivery of the 
non-membrane-permeative antibiotic gentamicin into mammalian cells by using Shigella flexneri membrane vesicles. Antimicrol Agents Chemother 42, 1476-1483.

Kadurugamuwa, J. L., Rohde, M., Wehland, J. \& Timmis, K. N. (1991). Intercellular spread of Shigella flexneri through a monolayer mediated by membranous protrusions and associated with reorganization of cytoskeletal protein vinculin. Infect Immun 59, $3463-3471$.

Kadurugamuwa, J. L., Lam, J. S. \& Beveridge, T. J. (1993). Interaction of gentamicin with the $\mathrm{A}$ band and $\mathrm{B}$ band lipopolysaccharides of Pseudomonas aeruginosa and its possible lethal effect. Antimicrob Agents Chemother 37, 715-721.

Kadurugamuwa, J. L., Mayer, A., Messner, P., Sára, M., Sleytr, U. B. \& Beveridge, T. J. (1998). S-layered Aneurinibacillus and Bacillus spp. are susceptible to the lytic action of Pseudomonas aeruginosa membrane vesicles. J Bacteriol 180, 2306-2311.

Kondo, K., Takade, A. \& Amako, K. (1993). Release of outer membrane vesicles from Vibrio cholerae and Vibrio parahaemolyticus. Microbiol lmmunol 37, 149-152.

Lakshminarayanaiah, N. (1984). Equations of Membrane Biophysics, pp. 7-64. New York: Academic Press.

Lauffer, M. A. (1989). Motion in Biological Systems, pp. 189-220. New York: Alan R. Liss.

Laukkanen, M.-L., Teeri, T. T. \& Keinanen, K. (1993). Lipid-tagged antibodies: bacterial expression and characterization of a lipoprotein-single-chain antibody fusion protein. Protein Eng 6, 449-454.

Levine, M. M., Ferreccio, C., Black, R. E., Tacker, O., Germanier, R. \& the Chilean Typhoid Committee (1989). Progress in vaccines against typhoid fever. Rev Infect Dis 11, S552-S567.

Li, Z., Clarke, A. J. \& Beveridge, T. J. (1996). A major autolysin of Pseudomonas aeruginosa, its subcellular distribution, its potential role in cell growth and division, and its secretion in surface membrane vesicles. J Bacteriol 178, 2479-2488.

Li, Z., Clarke, A. J. \& Beveridge, T. J. (1998). Gram-negative bacteria produce membrane vesicles which are capable of killing other bacteria. J Bacteriol 180, 5478-5483.

Lindberg, A. A. (1990). Aromatic-dependent Shigella strains as live vaccines. In New Generation Vacines, pp. 667-687. Edited by G. C. Woodrow \& M. M. Levine. New York: Marcel Dekker.

Mahan, M. J., Slauch, J. M. \& Mekalanos, J. J. (1992). Selection of bacterial virulence genes that are specifically induced in host tissues. Science 259, 686-688.
Maskell, D. J., Sweeney, K., O'Callaghan, D., Hormaeche, C. E., Liew, F. H. \& Dougan, G. (1987). Salmonella typhimurium aroA mutants as carriers of the Escherichia coli heat-labile enterotoxin $B$ subunit to the murine secretory and systemic immune systems. Microb Pathog 2, 211-221.

Mayrand, D. \& Holt, S. C. (1988). Biological activities of outer membrane vesicles. Can J Microbiol 35, 607-613.

Mouritsen, O. G. \& Bloom, M. (1993). Models of lipid-protein interactions in membranes. Annu Rev Biophys Biomol Struct 22, 145-171.

Roberts, M., Chatfield, S. N. \& Dougan, G. (1994). Salmonella as carriers of heterologous antigens. In Novel Delivery Systems for Oral Vaccines, pp. 27-58. Edited by D. T. O'Hagen. Boca Raton, FL: CRC Press.

Sansonetti, P. J. \& Arondel, J. (1989). Construction and evaluation of a double mutant of Shigella flexneri as a candidate for oral vaccination against shigellosis. Vaccine 7, 443-450.

Stocker, B. A. D. (1990). Aromatic-dependent Salmonella as live vaccine presenters of foreign epitopes as inserts in flagellin. Res Microbiol 141, 787-796.

Su, G.-F., Brahambhatt, H. N., Wehland, J., Rohde, M. \& Timmis, K. N. (1992). Construction of stable LamB-Shiga toxin B subunit hybrids; analysis of expression in Salmonella typhimurium aroA strains and stimulation of $B$ subunit-specific mucosal and serum antibody responses. Infect Immun 60, 3345-3359.

Wai, S. N., Takade, A. \& Amako, K. (1995). The release of outer membrane vesicles from the strains of enterotoxigenic Escherichia coli. Microbiol Immunol 39, 451-456.

Whitmire, W. M. \& Garon, C. F. (1993). Specific and nonspecific responses of murine $\mathrm{B}$ cells to membrane blebs of Borrelia burgdorferi. Infect Immun 61, 1460-1467.

Wispelwey, B., Hansen, E. J. \& Scheld, M. (1989). Haemophilus influenzae outer membrane vesicle-induced blood-brain barrier permeability during experimental meningitis. Infect Immun 57, $2559-2562$.

Zhou, L., Srisatjaluk, R., Justus, D. E. \& Doyle, R. J. (1998). On the origin of membrane vesicles in gram-negative bacteria. FEMS Microbiol Lett 163, 223-228.

Zimmerberg, J., Vogel, S.S. \& Chernomodik, L. V. (1993). Mechanisms of membrane fusion. Annu Rev Biophys Biomol Struct 22, 433-466.

Received 15 March 1999; accepted 13 April 1999. 